\title{
Analysis of refrigeration and heating efficiency of ground source heat pump
}

\author{
Hao $\mathrm{Wu}^{1}$, Wanfu Liu ${ }^{1, *}$, Wuqin $\mathrm{Qi}^{1}$ and Shuo $\mathrm{Ma}^{1}$ \\ ${ }^{1}$ School of Mechanical Engineering, Tianjin University of Commerce, Tianjin, China
}

\begin{abstract}
This paper studies the efficiency of ground source heat pump(GSHP) used in refrigeration and heating, comparing it with air source heat pump (ASHP) by means of theoretical analysis and experiment. The results were as follows, the cooling efficiency of GSHP in summer is higher than that of heating in winter because of the heat is transferred from high temperature to low temperature during cooling in summer, which can be compared to heat pipe; By calculating the power consumption of pump and fan, it is found that theoretically GSHP unit and system have higher heating efficiency than ASHP; The experiment shows that the daily average COP of the unit and the system is 4.22-4.62 and 3.26-3.55, respectively, both the unit COP and system COP of GSHP is higher than that of ASHP, which is consistent with the theoretical analysis.
\end{abstract}

\section{Introduction}

The weather in northern China is very cold in winter, and northern residents need a lot of heating in winter. In recent years, the heat pump heating methods represented by ASHP and GSHP have been greatly developed with the vigorous promotion of coal to electricity in rural areas $^{[1]}$. Energy conservation and environmental protection have been greatly improved, but there are still some problems. For example, ASHP is prone to frost formation on the surface of outdoor heat exchangers in in the northern cold area, which affects the heat exchange performance of the unit ${ }^{[2]}$. Although GSHP has no such phenomenon, the water pump of its cooling and heating system consumes a large amount of power and has different energy saving benefits.

Many scholars have studied the efficiency of GSHP refrigeration and heating system and ompared it with ASHP.

XiaohuiShe,LinCong,BinjianNie ${ }^{[3]}$ studied the method to improve the efficiency of VCRs, it was found that the condensation temperature of the system was reduced by $5^{\circ} \mathrm{C}$, and the cop of VCRs was increased by $14 \%$ when GSHP was used. ShuyangZhang, LunZhang, HongyangWei ${ }^{[4]}$ tested and analyzed the performance of GSHP in hot summer and cold winter area. The results show that the weighted average Coefficient of Performance of the heat pumps and system Energy Efficiency Ratio varied from 3.36 to 5.94 and 1.95 to 4.35 , respectively. Chan $\mathrm{Xia}$ and $\mathrm{Zhen} \mathrm{Bu}^{[5]}$ recorded and studied the actual working effect of the GSHP system in a townhouse in winter, and found that the COP of the heat pump units on weekdays and non-weekdays was 3.4 and 3.5, and the system COPs was 2.5 and 2.6. Ziqiang Liu, Jianhui Wang ${ }^{[6]}$ built a set of auxiliary solar heating system for GSHP. When the ASHP operated independently, the COP and system of the unit could reach above 7.5 and 6.1 , and the COP value decreased to 5.0 and 4.0 over time.

FrancescoTinti,AlbertoBarbaresi ${ }^{[7]}$ evaluated the efficiency of hybrid geothermal basket/ASHP on a case study winery based on experimental data, It is found that the seasonal performance coefficient of GSHP is 3.2, and the seasonal performance coefficient of refrigeration is 4.7. The cooling efficiency in summer is higher than that in winter. X.Q.Zhai, X.W.Cheng, R.Z.Wang ${ }^{[8]}$ studied Heating and cooling efficiency of a minitype GSHP system. As a result, The COP of the GSHP system was 3 in winter, The COP of the GSHP system was 3.2 in summer. Hanbao Zhu and Yasu Zhou ${ }^{[9]}$ studied the GSHP and found that the COP value of the GSHP was 4.200-4.800 under the refrigeration condition, and 3.900-4.550 under the heating condition.

According to the above research results, the working efficiency of GSHP is inconsistent, and the cooling efficiency of GSHP in summer is better than the heating efficiency in winter. Therefore, the efficiency of cooling and heating of GSHP is analyzed theoretically and compared with that of ASHP, what's more, set the GSHP heating test-bed to measure the heating efficiency, in order to provide some reference for the application of GSHP heating in cold areas.

$\overline{{ }^{*} \text { Wanfu Liu: wu_peihao0704@163.com }}$ 


\section{Theoretical analysis and calculation}

\subsection{The theoretical analysis}

Regardless of ASHP or GSHP, the working medium cycle of the unit is the Rankine cycle, its cycle T-S can be referred to Figure 1. The cycle is $1-2-3-4-5-1^{[10]}$.

Figure 1 is the T-S diagram of working medium cycle of heat pump during summer cooling, a represents ASHP, b represents GSHP.

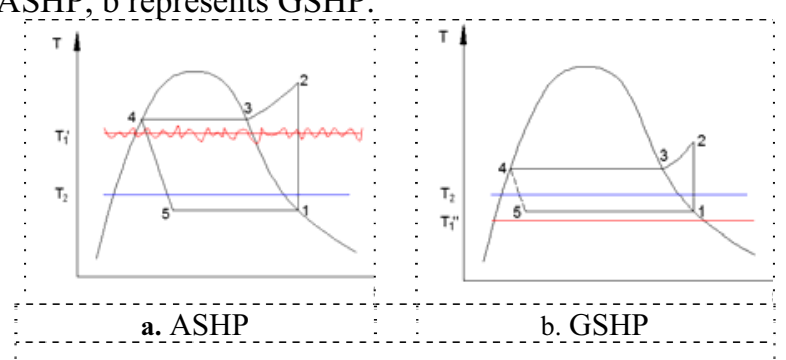

Figure 1. T-s diagram of working medium cycle of heat pump during summer cooling

In the figure 1 , the wavy line represents the outdoor air temperature in summer, $\mathrm{T}_{1}{ }^{\prime}$ is the outdoor ambient temperature in summer, $\mathrm{T}_{1}{ }^{\prime \prime}$ is the underground soil temperature in summer, and $T_{2}$ represents the indoor temperature in summer.

The refrigeration coefficient of heat pump can be expressed as:

$$
\varepsilon=\frac{q_{c}}{w_{n e t}}
$$

Where: $q_{c}$-Heat absorbed in one cycle of heat pump working medium cycle, kw; $w_{n e t}$-The compressor power consumption, kw;

Under the same refrigeration requirements, the cooling capacity and evaporation temperature of the ASHP unit and the GSHP unit remain unchanged. As the underground soil temperature is lower than the outside air temperature in summer, the condensing temperature of the working medium of the GSHP unit is lower than the condensing temperature of the working medium of the ASHP unit, the compressor of GSHP unit has lower exhaust pressure, lower power consumption and higher efficiency.

Figure 2 is the T-S diagram of heat pump working medium under the working condition of winter heating.

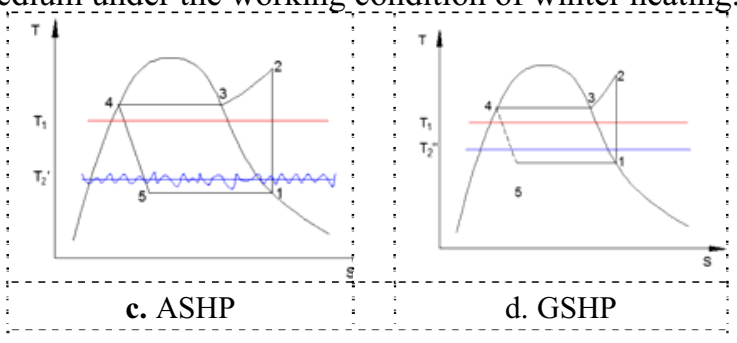

Figure 2. T-s diagram of working medium cycle of heat pump during winter heating

$\mathrm{T}_{1}$ represents the indoor temperature, the wavy line represents the outdoor air temperature in winter, $\mathrm{T}_{2}{ }^{\prime}$ represents the average outdoor air temperature in winter, and $\mathrm{T}_{2}$ " represents the underground soil temperature in winter. The underground soil temperature is stable all year round and higher than the outside air temperature in winter.

The heating coefficient of heat pump can be expressed as:

$$
\varepsilon^{\prime}=\frac{q_{e}}{w_{\text {net }}}
$$

Where: $q_{e}$-Heat released in one cycle of heat pump working medium cycle, kw; $w_{n e t}$-The compressor power consumption, kw;

Under the Same heating requirements, the heating capacity and condensation temperature of the air source heat pump unit and the GSHP unit remain unchanged. As the underground soil temperature is higher than the outside air temperature in winter, the evaporation temperature of the working medium of the GSHP unit is higher than the evaporation temperature of the working medium of the ASHP unit, the compressor of GSHP unit has higher suction pressure, lower power consumption and higher efficiency.

According to the above analysis, the energy saving potential of GSHP is much greater than that of ASHP.

When the GSHP is used for cooling in summer, it can be compared to a heat pipe. The working principle of the heat pipe is shown in Figure 3.

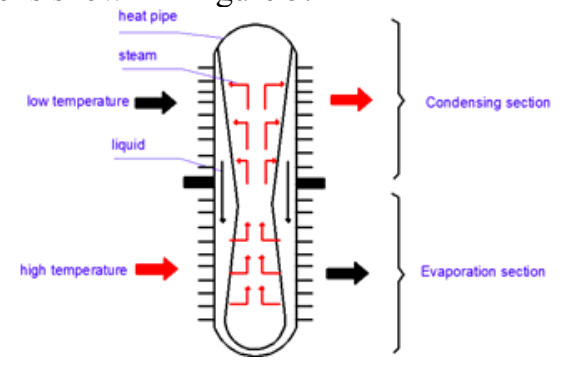

Figure 3. Working principle diagram of heat pipe

Heat pipe makes full use of the principle of heat conduction and the rapid heat transfer property of phase change medium. The working medium evaporates at high temperature and condenses at low temperature, bringing heat quickly and efficiently from high temperature to low temperature.

In cold areas, the indoor set temperature is $22^{\circ} \mathrm{C} \sim 28^{\circ} \mathrm{C}$ in winter, the underground soil temperature in northern China is generally around $17^{\circ} \mathrm{C}$, lower than the indoor set temperature. When GSHP is used for refrigeration, the heat transfer direction is from high temperature to low temperature, and its working principle is equivalent to the heat pipe, which consumes less power and has higher efficiency. Therefore, the cooling efficiency of GSHP in summer is higher than that of heating in winter.

The above analysis does not consider the power consumption of other components except the compressor, In the actual work process, in addition to the compressor power consumption, the ASHP system power consumption also includes the fan power consumption at the indoor heat exchanger and outdoor heat exchanger. The GSHP system power consumption includes the 
water pump power consumption on the Source side and the Terminal side, and the fan power consumption of the fan coil unit on the Terminal side. Let's take heat pump heating in winter as an example for analysis.

When heat pump is used for heating, its system operating efficiency (COP) is expressed as:

$$
\begin{gathered}
C O P_{a}=\frac{Q_{e a}}{P_{c a}+P_{f 1 a}+P_{f 2 a}} \\
C O P_{g}=\frac{Q_{e g}}{P_{c g}+P_{f 1 g}+P_{p 1}+P_{p 2}}
\end{gathered}
$$

Where:

Subscript: $a$-ASHP; $g$ - GSHP; $e-$ Condenser; $c$ - co mpressor; $f$-Fan; $p$-Water pump; 1 -Source side; 2-Terminal side

COP - Heating efficiency of heat pump system; $P$-Power, kw $/ \mathrm{h} ; \quad Q$ - Heat exchange capacity of heat exchanger per hour, kw;

Under the same heating conditions, $Q_{e a}=Q_{e g}$, $P_{f 1 a}=P_{f 1 g}$, GSHP heating system is required to save energy than ASHP heating system, $C O P_{g}>C O P_{a}$, $P_{p 1}+P_{p 2}-P_{f 2 a}<P_{c a}-P_{c g}$

\subsection{The theoretical calculation}

The fan power is calculated according to the following formula

$$
P=L p /\left(3600 \times 1000 \eta_{0} \eta_{1}\right)
$$

Where: $P$-Fan power consumption, kw; $L$-Air volume, $\mathrm{m}^{3} / \mathrm{h} ; \quad p$-Full wind pressure of the fan, $\mathrm{Pa}$; $\eta_{0}$-Internal efficiency of the fan, generally $0.75 \sim 0.85$; $\eta_{1}$-Mechanical efficiency, $0.95 \sim 0.98$;

The air volume and Full wind pressure of the fan can be estimated according to "Heat Exchanger Principles and Design"[11], which will not be repeated here.

The fan power consumption expression is as follows:

$$
P=\frac{Q}{\rho c_{p} \Delta t}\left(5.1 v_{N F}^{1.504} N \Phi_{f}+\Delta p_{2}\right) /\left(1000 \eta_{0} \eta_{1}\right)
$$

The fan power consumption is estimated as:

$$
P_{f} \approx 1.32 \times 10^{-3} Q \sim 2.17 \times 10^{-3} Q
$$

Obtain:

$$
\begin{aligned}
& P_{f 1} \approx 1.32 \times 10^{-3} Q_{c} \sim 2.17 \times 10^{-3} Q_{c} \\
& P_{f 2} \approx 1.32 \times 10^{-3} Q_{e} \sim 2.17 \times 10^{-3} Q_{e}
\end{aligned}
$$

The calculation formula of water pump power consumption is as follows:

$$
P=W / \eta=\rho L g H / \eta=g L H / 3600 \eta
$$

Where: $P$-Water pump power, $\mathrm{kw} / \mathrm{h} ; W$-Effective power of water pump, $\mathrm{kw} / \mathrm{h}$; $\eta$-Water pump efficiency, generally $0.75 \sim 0.85$; $\rho$-The density of water, $1000 \mathrm{~kg} / \mathrm{m}^{3} ; L$ - Water pump flow, $\mathrm{m}^{3} / \mathrm{h} ; g$-Acceleration of gravity, $9.81 \mathrm{~N} / \mathrm{Kg}$; $H$-Water pump Lift, m;

$$
L=\frac{Q}{c \rho \Delta T}(1.1 \sim 1.2)=\frac{Q}{1.163 \Delta T}(1.1 \sim 1.2)
$$

Where: $Q$ - Heat exchange capacity between water pump and heat exchanger, $\mathrm{kw} ; c$-Specific heat capacity of water, $4.2 \mathrm{~kJ} / \mathrm{kg} ; \Delta T$-System temperature between supply and return water, generally $4.5 \sim 5^{\circ} \mathrm{C}$;

According to the formula (10), (11)

$$
P=\frac{Q}{1.163 \Delta T}(1.1 \sim 1.2) g H / 3600 \eta
$$

The power consumption of the water pump is estimated as follows:

$$
P_{p} \approx 6.4 \times 10^{-4} H Q
$$

Obtain:

$$
\begin{gathered}
P_{p 1} \approx 6.4 \times 10^{-4} H_{1} Q_{c} \\
P_{p 2} \approx 6.4 \times 10^{-4} H_{2} Q_{e}
\end{gathered}
$$

Where: $H_{1}-$ Water pump Lift at ground source side, m; $H_{2}$-Water pump Lift at Terminal side, m;

Now simulating an ordinary rural residential building is heated in winter. The building type is three rooms, one hall, one kitchen and one bathroom, the height of the building is $3 \mathrm{~m}$ and the air conditioning area of the building is $200 \mathrm{~m}^{2}$. According to "Design Code for Heating ventilation of Civil Building and Air Conditioning" and "Energy saving design standard for rural residential building", the indoor temperature is set at $22^{\circ} \mathrm{C}$, and the building heat load is $12 \mathrm{KW}$ per hour in winter.

The heating efficiency and the power consumption of each component of ASHP system and GSHP are estimated by REFRIGERATION and formula (8) (9) (14) (15), Its value are shown in Table 1. Among them, the lift of water pump at the ground source side and the terminal side were assumed to be $30 \mathrm{~m}$ and $15 \mathrm{~m}$ respectively, fan power consumption is assumed to be maximum.

Table 1. Efficiency and power consumption of each component of GSHP and ASHP

\begin{tabular}{|c|c|c|c|c|}
\hline $\mathrm{P}_{\mathrm{p} 1}$ & $\mathrm{P}_{\mathrm{p} 2}$ & $\mathrm{P}_{\mathrm{fla}}=\mathrm{P}_{\mathrm{f} 1 \mathrm{~g}}$ & $\mathrm{P}_{\mathrm{f} 2 \mathrm{a}}$ & $\mathrm{P}_{\mathrm{ca}}$ \\
\hline 0.202 & 0.106 & 0.027 & 0.022 & 1.36 \\
\hline $\mathrm{P}_{\mathrm{cg}}$ & $\mathrm{P}_{\mathrm{p} 1}+\mathrm{P}_{\mathrm{p} 2}-\mathrm{P}_{\mathrm{f} 2 \mathrm{a}}$ & $\mathrm{P}_{\mathrm{ca}}-\mathrm{P}_{\mathrm{cg}}$ & $\mathrm{COP}_{\mathrm{a}}$ & $\mathrm{COP}_{\mathrm{g}}$ \\
\hline 0.77 & 0.286 & 0.483 & 8.5 & 10.8 \\
\hline
\end{tabular}

As can be seen from Table 1, $P_{p 1}+P_{p 2}-P_{f 2 a}<P_{c a}-P_{c g}$, the GSHP heating system works more efficiently, $C O P_{g}>C O P_{a}$.

\section{Experimental analysis}

In this paper, set up GSHP heating test-bed in the Tianjin University of Commerce. seting six indoor temperature working conditions $\left(18^{\circ} \mathrm{C}, 20^{\circ} \mathrm{C}, 22^{\circ} \mathrm{C}, 24^{\circ} \mathrm{C}, 26^{\circ} \mathrm{C}\right.$ and $28^{\circ} \mathrm{C}$ ) in winter to analyze the changes of GSHP unit and system heating efficiency (COP) under different room seting temperature. The GSHP heating test-bed consists of vertical double U-tube buried heat exchanger, water 
pump at ground source side, GSHP unit, fan coil unit, water pump at at terminal side and data acquisition system. The system connection diagram is shown in Figure 4.

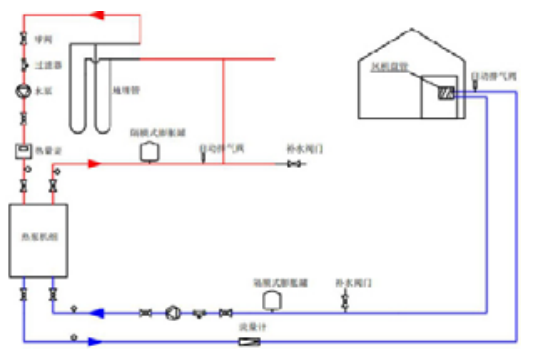

Figure 4. Connection diagram of GSHP heating system

Figure 5 shows the unit average COP and the system average COP of GSHP at different room seting temperature.

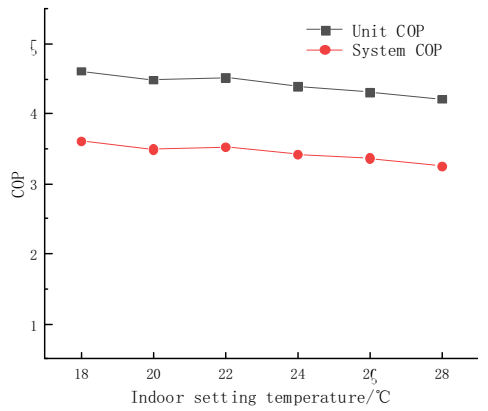

Figure 5. Daily average COP of system and unit at different room temperature

As can be seen from the figure 5 , under various room temperature conditions, the daily average COP of the unit was above 4.22 and the highest was 4.62 . The daily average COP of the system was slightly lower, the highest was 3.55 and the lowest was 3.26. There is a big difference between the experimental data and the theoretical data because of the isentropic efficiency of compressor and heat loss in actual operation. However, the heating efficiency of GSHP is much higher than that of air-con and ASHP in daily use, considering the power consumption of water pump and fan, its heating efficiency is greatly reduced, but it still has slight advantages. which is consistent with the theoretical calculation.

\section{Conclusion}

The working principle of GSHP used for refrigeration in summer is similar to that of heat pipe, Its heat transfer direction is from high temperature to low temperature, so its cooling efficiency in summer is higher than heating efficiency in winter.

In theory, the energy saving potential of GSHP for cooling and heating is greater than that of ASHP, water pump, fan power consumption is as follows: $P_{p} \approx 6.4 \times 10^{-4} \mathrm{HQ} \quad, \quad P_{f} \approx 1.32 \times 10^{-3} Q \sim 2.17 \times 10^{-3} Q \quad$, considering the power consumption of water pump and fan, COP of GSHP heating system is still greater than that of ASHP heating system.

under various room temperature conditions, the daily average COP of the unit and the system of GSHP is 4.22-4.62 and 3.26-3.55, respectively. Experimental result is lower than the theoretical value, but the unit COP of GSHP is much higher than that of the air-con and ASHP used in daily life, although the system COP is low, it still has a slight advantage, which is consistent with the theoretical calculation.

\section{References}

1. J. Zhao, X. Gong, P. Sun, F. Zhao, G. Liu, Comparison of heating forms in rural areas of North China, Energy Conservation, 38(2019),pp. 161-164

2. P. Wang, J. Dong, J. Dong, Discussion on defrosting Technology of Air source heat pump Unit, Heating Ventilating \& Air Conditioning, 40(2010), pp. 123-128

3. X. She, L. Cong, B. Nie, G. Leng, H. Peng, ergy-efficient and -economic technologies for air conditioning with vapor compression refrigeration: A comprehensive review, Applied Energy, 232(2018), pp.157-186

4. S. Zhang, L. Zhang, H. Wei, J. Jing, Field testing and performance analyses of ground source heat pump systems for residential applications in Hot Summer and Cold Winter area in China, Energy and Buildings, 133(2016), pp. 615-627

5. C. Xia, Z. Bu, Field measurement and analysis of ground source heat pump system in a townhouse in Shanghai in winter, Shanghai Energy Conservation, (2016), pp.247-251

6. Z. Liu, J. Wang, W. Liu, G. Peng. Experimental study on heating operation of composite ground source heat pump system in winter, Renewable Energy Resources, (2007), pp. 47-50

7. Francesco Tinti, Alberto Barbaresi, Daniele Torreggiani, Davide Brunelli, Evaluation of efficiency of hybrid geothermal basket/air heat pump on a case study winery based on experimental data, Energy and Buildings, 151(2017), pp. 365-380

8. X.Q. Zhai, X.W. Cheng, R.Z. Wang, Heating and cooling performance of a minitype ground source heat pump system, Applied Thermal Engineering, 111(2017), pp. 1366-1370

9. H. Zhu, Y. Zhou, Technical and economic performance comparison of ground source heat pump and air-cooled heat pump, Renewable Energy Resources, (2006), pp. 86-89

10. W. Shen, J. Tong, Engineering Thermodynamics, Beijing: Higher Education Press, (2016), pp. 361-362

11. M. Shi, Z. Wang, Heat exchanger principle and design, Southeast University Press, (2014), pp. 180-182 